\title{
Active Control of Sound Radiation by Structures Using Near-field Sensing Strategies
}

\author{
Xun Li, Xiaojun Qiu ${ }^{\dagger}$ and Colin H. Hansen ${ }^{\dagger}$ \\ Department of Mechanical Engineering, Adelaide University, South Australia 5005, Australia
}

(Received 8 January 2001; accepted 11 January 2001)

\begin{abstract}
The active control of sound radiated from structures using two near-field sensing strategies is investigated theoretically. The two sensing strategies involve minimising the sum of the sound intensities and minimising the sum of the squared sound pressures in the acoustic field close to the surface of a rectangular panel. A new quadratic expression is derived for the minimisation of the sum of the sound intensities in the near-field. The formulation is expressed in terms of the primary sound field and the measured or calculated transfer functions from the control inputs to the error sensor outputs. Thus, the expression given here can be used to solve any complex problem where the control source to error sensor transfer functions and primary sound field pressures can be determined. In this example, a flat panel is modelled with a harmonic point force excitation and several point force control sources. To compare the control performance achieved by the proposed near-field sensing strategies, the radiated sound field is minimised in the near-field, and then the control results are evaluated in the far-field for each test case.
\end{abstract}

${ }^{\dagger}$ Member of the International Institute of Acoustics and Vibration (IIAV)

\section{INTRODUCTION}

In the field of active control of sound radiated from structures, only limited previous work ${ }^{1-7}$ has been undertaken on the minimisation of the radiated sound using near-field error sensing of sound intensity. In the work of McLoughlin et al., ${ }^{1}$ the minimisation of the noise radiated by a small transformer (7.5 MVA) was achieved with an average reduction of the 120 and $240 \mathrm{~Hz}$ tones of $10-15 \mathrm{~dB}$ by means of near-field sensing of the sound intensity. However, no theoretical analysis was provided. Wang and Fuller ${ }^{2}$ reported a theoretical study of the near-field pressure and intensity distributions of actively controlled panel-radiated sound. In their work, a control system consisted of a single control force and a single error sensor. It was found that, although limited to the 3,1 mode resonance case, applying control leads to an overall reduction in the magnitudes of the near-field pressure and intensity vectors, while at the same time the complexity of the sound radiation pattern is increased. Qiu et al. ${ }^{3}$ presented a theoretical comparison of several near-field error sensing strategies for active control of harmonic free-field sound radiation. In their work, sensing strategies included the minimisation of the acoustic potential energy density, acoustic kinetic energy density, total acoustic energy density and mean active sound intensity. They concluded that the most appropriate near-field error sensing strategy is to minimise the sum of the mean active sound intensities provided that a large number of error sensors are used.

Lee and Park $^{4}$ reported on the evaluation of near-field sensing strategies for the control of noise radiated by a fluidloaded rectangular plate. For comparison, a total of four active control strategies were involved in their study: 1) the integral of the near-field surface sound intensity over the plate surface; 2) the integral of the squared sound pressure over a hemisphere in the far field; 3 ) the integral of the near-field surface reactive intensity over the plate and 4) the integral of the squared velocity over the plate surface. The results from numerical simulations showed that for the fluid-loaded plate, the optimal result achieved by minimising the radiated sound power in the near-field was quite similar to that achieved by minimising the sound power in the far-field. Also the control result obtained by the minimisation of the reactive power was almost the same as that obtained by the minimisation of the vibration energy. The sound power level reduction achieved by minimising the vibration energy was much lower than that obtained using near-field active intensity minimisation. This is because minimising the plate vibration level led to the reduction of the near-field pressure level but resulted in less sound power level reduction in the far-field. However, the sound pressure in the near-field consists of a combination of a propagating energy component and non-propagating energy component, and therefore reducing the near-field sound pressure may not necessarily minimise the sound power in the far-field.

Recently, work has been reported ${ }^{5-8}$ on minimising sound radiated from structures using near-field sensing strategies, i.e., the minimisation of the sum of the squared sound pressures and the minimisation of the sum of the sound intensities in the near-field. The authors concluded that far field sound reduction was achievable by minimising the sound intensity in the near-field, but a large number of sensors was required.

This paper is concerned with a theoretical analysis of active control of the sound radiated from planar structures using near-field sensing strategies and the effect of various parameters, such as sensing array density and distance from the structure, on the result achieved. The sensing strategies are the minimisation of the sum of the squared sound pressures and the minimisation of the sum of the sound intensities in the near-field. For calculating the sound intensity, the two microphone technique is employed. To evaluate the effect of the noise source dimension on the control performance, the noise source is defined as a small source when $\kappa \ll \pi$ and as an extended source when $\kappa>\pi$ where the normalised source 